\title{
Magnetic resonance imaging of pudendal nerve: technique and results
}

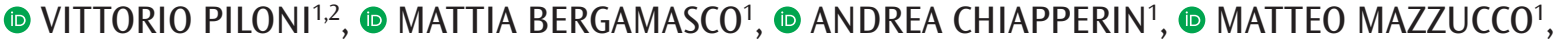 \\ (1) TOMMASO FELICI ${ }^{2}$, (D) JESSICA ANDREATINI², (1) NOEMI NUCERA ${ }^{2}$, (1) ELENA FREDDI ${ }^{2}$ \\ ${ }^{1}$ Affidea-Iniziativa Medica, Padua, Italy \\ 2Diagnostica Marche, Ancona, Italy
}

\section{ABSTRACT}

Objective: To update the ongoing contribution of magnetic resonance imaging (MRI) in clinical practice

Materials and Methods: The imaging series of pelvic MRI examinations carried out in 580 consecutive patients (401 women, 179 men, mean age $51 \pm 5.3$ year and $40 \pm 4.4$ year, range: 18-87 and 24.62 year, respectively) in two different diagnostic centres from March 2012 (centre A) and April 2017 (centre B) to October 2020 in patients with chronic pelvic pain, were retrospectively reviewed for evidence of features of pudendal nerve injury. Ten volunteer asymptomatic nulliparous females (mean age: $24 \pm 2.1$ years, range: 23-27 years) were also included as control group.

Results: Pudendal nerve injury could consistently be documented on specific MR pulse sequences as hyperintensity and distortion of the nerve pathway in up to $454(78.27 \%)$ of patients and in none of the control group, indicating the high reliability of the diagnostic criteria and leading to implementation of an established MR imaging protocol.

Conclusion: MRI is promising for clinical practice and research in pudendal neuropathy.

Keywords: MR-neurography; chronic pelvic pain syndromes; pudendal nerve entrapment

\section{INTRODUCTION}

Since its first description in 1992 and subsequent years, ${ }^{1-4}$ magnetic resonance imaging (MRI) of pudendal nerve has rapidly gained wide acceptance by the medical community as a valuable tool for decision-making and therapy planning in patients with chronic pelvic pain syndromes. ${ }^{5}$ The term "MRneurography" has come into practical use to describe the direct depiction of nerves in the body using special modifications of the standard MR imaging technique which allow detection of the signal arising from inside the nerve itself rather than from the surrounding tissues. More specifically, given the intraneural source of the signal, the images obtained during the examination were thought to provide important information about the internal state of the nerve such as the presence of irritation, nerve swelling, compression, pinch or injury. From the technical point of view, the basic principle for a successful examination includes suppression of the signal coming from the bright fat, so as to better depict the intrinsic signal from nerve water as a hyperintense linear structure. In addition, thanks to recent advances in technology, it has become possible to obtain also contemporary suppression of the signal of similar-appearing surroundings structures, namely the blood in the vascular 
tree, ${ }^{6}$ thus eliminating any source of confusion during image interpretation. The current paper will describe the application and criteria for interpretation of a standardized MR-neurography examination which has been matured by us over a nine-year experience in two different imaging diagnostic centres in Italy.

\section{Imaging centres}

At the beginning of March 2012, the MR neurography examination was implemented at the Diagnostic Centre of Affidea- Iniziativa Medica of Monselice, Padua, Italy (center A); subsequently, since April 2017 the same protocol was also applied at the Diagnostica Marche Imaging Center of Osimo Stazione, Ancona, Italy (centre B). All examinations were developed on a 1.5 T, horizontally oriented superconductive scanner (Philips, Multiva model, model 2014, Netherland A; Siemens model 2014, Germany, center B). Although performed by two different technical staffs ( $M$ B , A C, MM, centre A; T F, J A, N N, E F, centre B), image analysis and interpretation were conducted by the same radiologist (VP).

\section{Patient population}

Overall, 580 consecutive patients (479 at centre A, 101 at centre B) were enrolled into the study. Of them, 401 were women and 179 men, mean age $51 \pm 5.3$ year and $40 \pm 4.4$ year, range: 18 87 and 24.62 year, respectively. Before imaging, all patients had had a complete physical examination by the referring physicians (urologists, gynecologists, colorectal surgeons, physiatricians, neurophysiologists, anaesthesists, osteopathes) which was considered mandatory in order to select the optimal imaging protocol in singular cases. Clues for examination included chronic pelvic pain arising from the pelvic floor district, involving the genital area, root of the thighs, groins and gluteal region, more or less associated with one of the following, alone or combined with each another: recurrent cystitis, non bacteric prostatitis, sexual intercourse, direct trauma on the pelvic region from accidental fall and intense sport activity (biking), prolonged sitting position at work, chronic strain at stool, prior pelvic colorectal or lower urinary tract surgery and obstetric trauma. In the sexually active female patients, the issue of vulvodynia received more in depth attention with regard to the characterization of presenting symptoms (see Table 1). More specifically, current findings, compared with those of ten asymptomatic young volunteers as control, are the basis of an ongoing study which has been carried out in search of obtaining better subdivision of symptoms in different categories and discovering any potential correlation with the diagnostic yield of MR imaging.

Patients were instructed to reach the diagnostic room after rectal cleansing with a disposable enema on the day before. The imaging day, during the interview with the radiologist they are

$\begin{aligned} & \text { Table 1. List of symptoms associated with vulvodynia in } \\
& \text { Thirty-five, consecutive sexually active female patients of } \\
& \text { the current study }\end{aligned}$
\begin{tabular}{|l|l|l|} 
Presenting symptom & n & $\%$ \\
\hline Pain at or after intercourse & 27 & 77.1 \\
\hline Exaggerate sensitivity (allodynia) & 23 & 65.7 \\
\hline Inability to wear tight-fitting clothes & 21 & 60 \\
\hline Pinprick & 18 & 51.4 \\
\hline Burning and increased urinary frequency & 17 & 48.5 \\
\hline Loss of sensation at penetration and of desire & 15 & 42.8 \\
\hline Electric discharge & 16 & 45.7 \\
\hline Spasm/fascicular contractions (vaginism) & 14 & 40.0 \\
\hline $\begin{array}{l}\text { Impatience with physical contact } \\
\text { with foreign objects of any kind }\end{array}$ & 12 & 34.2 \\
\hline Change in bowel habit & 8 & 22.8 \\
\hline n: Number & & \\
\hline
\end{tabular}

helped to fill a specific history-taking form, developed to collect data on symptoms onset, duration, characteristics, irradiations, either aggravating or relieving factors, and adverse impact on voiding, evacuation and sexual activity, if any. Finally, just before starting the examination, patients are asked to empty their bladder in the toilet adjacent to the diagnostic room.

\section{Image technique}

After continuous refinements of the technical setting with the help of the specialist technician of the two manufacturers (Philips and Siemens, respectively), MR imaging was standardized so as to minimize the acquisition time while optimizing the examination's image quality and diagnostic capabilities, i.e. the line sharpness, organ definition and conspicuity of pathology. In particular, during image acquisition, special attention is deserved to obtain correct patient positioning on the diagnostic table in order to avoid any asymmetry which might adversely affect image reading. In practice, with no need for intravenous contrast administration, the body pelvis was firstly depicted by a high-resolution T2-weighted turbo spin echo pulse sequence in the axial, sagittal and coronal planes in all subjects, as reported in previous articles. ${ }^{7}$ Optimal visualization of the anatomy in its entirety, including pelvic organs, neural pathways, fat recesses, muscles, fascia and ligaments was considered an integral part of the examination and of utmost importance to rule out any abnormality acting as trigger point responsible of chronic pain syndrome. ${ }^{8,9}$ After this, a peripheral pulse device was applied on the $2^{\text {nd }}$ finger of the left hand for cardiac gating and pudendal nerve imaging, using the same scan planes. In practice, for evaluating the nerve free of flow and motion artifact formation, a preparatory short-inversion-time inversion-recovery (STIR) 
radio frequency pulse sequence is employed which selectively saturate spins located in regions outside the image volume. Such a cardiac-triggered STIR sequence, thanks to a segmented rapid acquisition with relaxation enhancement readout and inversion-recovery delay adjusted to null fat, eliminates signal from flowing blood. As result, by this technique the signal of fat and blood flow is suppressed while living intact that of the excess of water entrapped in the damaged nerve, thus leading to its direct visualization.

\section{Image analysis}

As clearly reported in the literature, basic criteria for the diagnosis of peripheral nerve neuropathy at MR imaging relies on evidence of increased signal intensity at the site of nerve damage, with or without presence of any anatomic distortion, entrapment and/or change in caliber. As such, after identification of the paths of the pudendal nerve along its entire course at the level of (1) greater and lesser sciatic foramens, (2) ischial spines and (3) Alcock canal, great relevance by the radiologist is deserved to the analysis of various muscles and vessels in the region of interest. The above in search of any change in their shape, morphology, thickness and signal intensity as potential source of nerve trapping (Figure $1 \mathrm{a}$ and b). Thereafter, the second and most important task, is to detect any sign of signal hyperintensity along the nerve pathway. For this, unlike what occurred at the beginning with our preliminary experience, ${ }^{7}$ routine use of the double inversion recovery fat sat and black blood pulse sequences technique, which selectively suppresses the signal of fat and flowing blood, proved critical when assigning the judgment of nerve damage with superior diagnostic confidence (Figure 2 and 3). As it is known, black blood pulse sequences are not affected as STIR sequences by the so called "magic angle artifact" which consists of an increase in signal intensity which occurs when nerve's collagen orientation to the constant magnetic induction field approaches $55^{\circ},{ }^{10}$ thus simulating disease.

\section{RESULTS}

Overall, despite the gender disproportion (69.1\% females, 30.9\% males), mean age and range of the population examined were not significantly different (mean age: $51 \pm 5.3$ year and $40 \pm 4.4$ year, range: $18-87$ and 24.62 year, respectively). Conversely, the total amount of examinations performed at centre A overtly exceeded that of centre B (479 vs 101). Regardless of the different duration of the diagnostic activity - almost 5 years longer at center $\mathrm{A}-\mathrm{a}$ possible explanation must be sought in the different economic cost for the patient, which was only 36.15 euros when covered by the National Health System and no more than 200 euros in case of lack of it at centre A, as opposed to 320

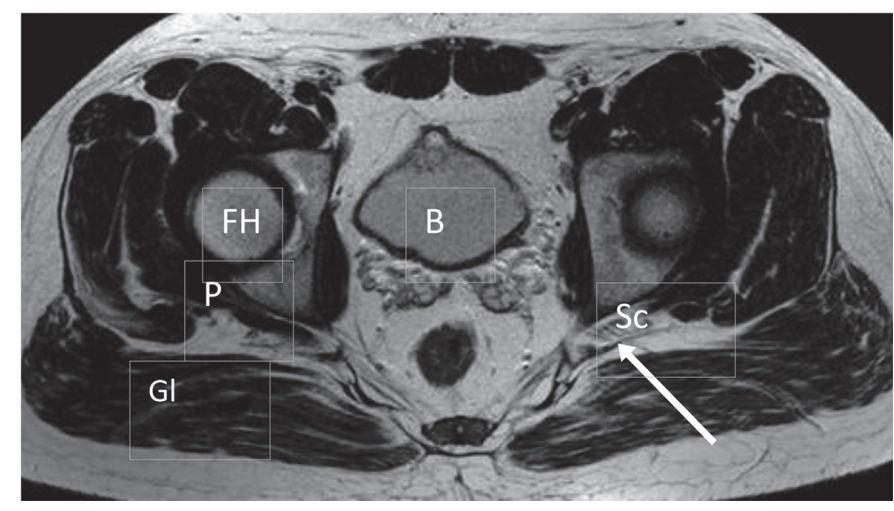

Figure 1a. T2 w TSE axial MRI of the pelvis of a forty nine yearold man with history of prior intense biking activity with chronic pain at the left gluteal region extended to the scrotal area and lower left leg: the left piriformis muscle is almost totally replaced by fat (arrow) with entrapment of sciatic and pudendal nerve at the exit of the lesser sciatic foramen TSE: Turbo spin echo, MRI: Magnetic resonance imaging, $\mathrm{FH}$ : Femoral head, Gl: Gluteus max muscle, B: Bladder, P: Piriformis muscle, Sc: Sciatic nerve

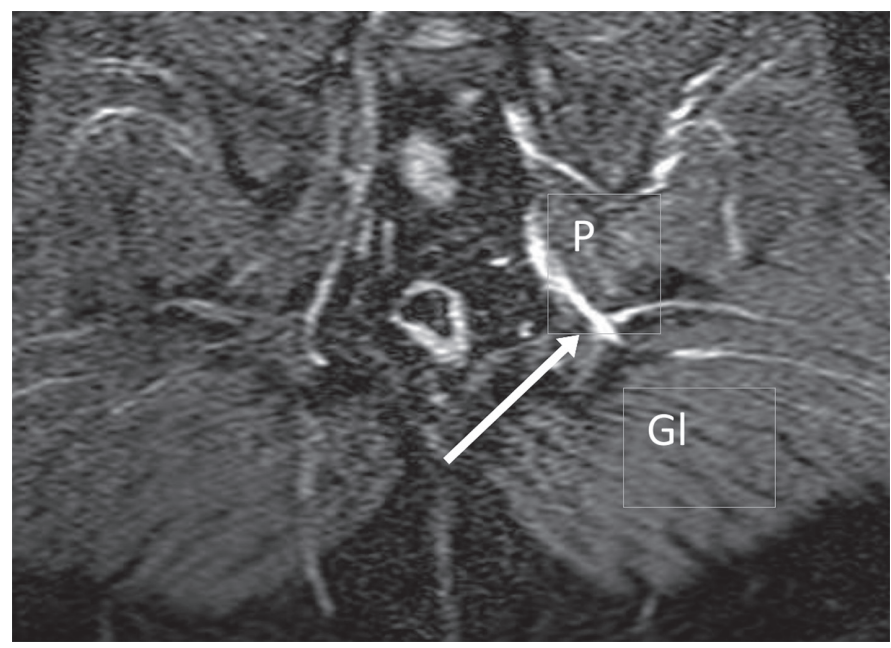

Figure 1b. On the coronal double IR FAT sat_BB pulse sequence the sciatic nerve (arrow) shows distortion, with increase of signal intensity and caliber

IR: Inversion-recovery, Gl: Gluteus max muscle, P: Piriformis muscle

euros at centre B, due to totally private assistance. Interestingly, however, the trend analysis (Figure 4) of pure quantitative activity reveals that the most striking difference between the two centres concerns the last two years, a period to be considered parallel. As such, different causes should be hypothesized, including the superior collaborative attitude of various referring physicians and their increased interest and curiosity toward any current advance in diagnostic technology, to name a few. Traditionally, both characteristics are known to be more pronounced in the North of Italy (centre A) where physicians keep particular attention to the issues of clinical and social impact resulting 


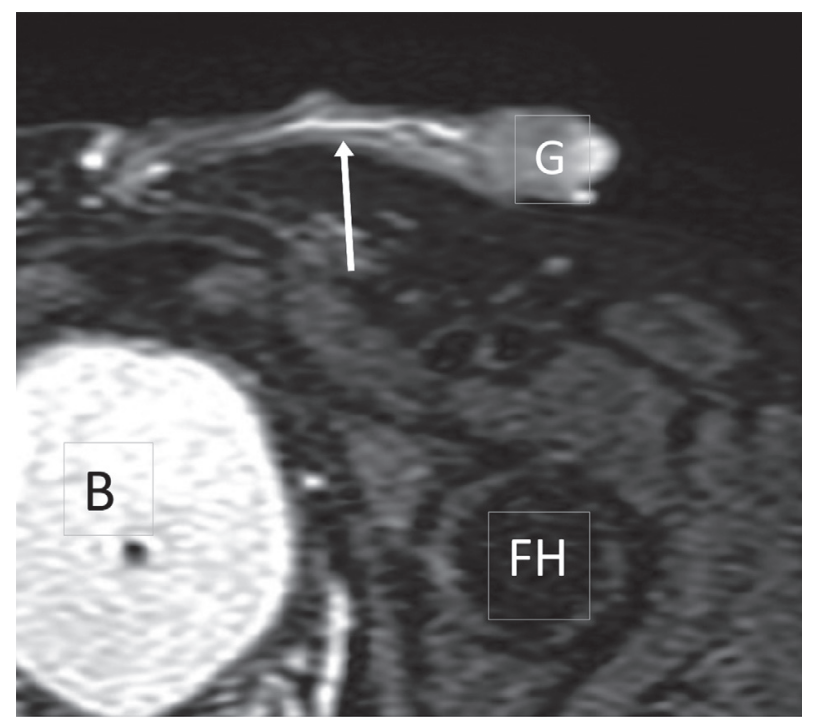

Figure 2. Thirty-four year-old man with LUTS and history of acute pain at intercourse, allodynia, pain in the region of corpus spongiosum of urethra and hyperemia of the navicular fossa of the penis. At MR neurography with pulse sequence, typical appearance of increased signal intensity was shown along the course of the dorsal nerve of penis (arrow)

LUT: lower urinary tract symptom, MR: Magnetic resonance, B: Bladder, FH: Femoral head, G: Glans of penis

from adopting any single medical action, namely that of costbenefit aspect associated with use of innovative technology. On the other hand, the analysis of the diagnostic yield of the examination has shown quite similar results in centre A and B, as follows: (1) Damage of pudendal nerve along the Alcock canal affecting the labial and clitoral branches (female) and scrotal or dorsal penis nerve (male), was the most frequent finding and accounted for 71 and $58 \%$ of cases vs 69 and 55\% at centre A and $B$, respectively; (2) Involvement of the gluteal and sciatic nerves at their encroachment with the greater or lesser sciatic foramen ranked second with 51 and $48 \%$ of cases vs 46 and 52\%, followed by (3) increased signal intensity of the nerve in close proximity of the ischial spine in 39 and $41 \%$ of cases vs 43 and $38 \%$, respectively.

The changes described above were not seen in anyone of the control group.

\section{CONCLUSIONS}

The expanding role of MRI places it at the side of neurophysiological tests for the assessment of peripheral nerve pathology. ${ }^{11}$ While neurophysiologic testing is required to differentiate between demyelination and axonal degeneration by measuring the electrical activity of nerves according to different parameters, such as latency, amplitude and conduction velocity, the application of MRI, allows direct visualisation of the

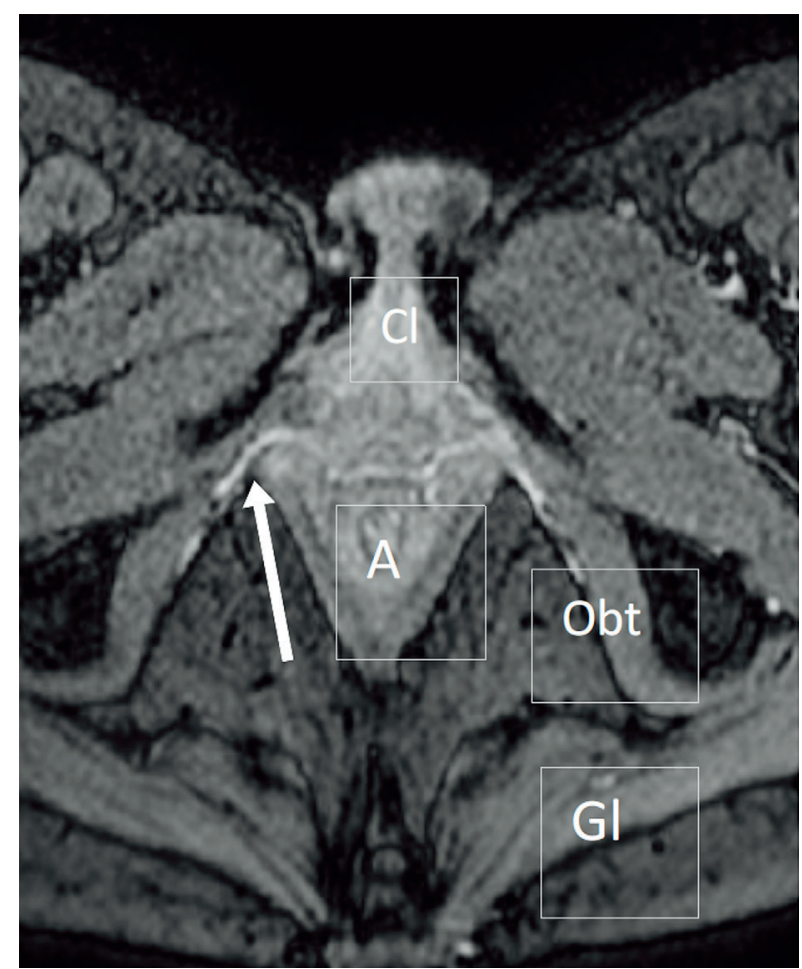

Figure 3. Forty year-old woman with long standing history of cystitis since childhood and more recent recurrent episodes of pain during intercourse foreplay, followed by urethral burning just after it. At T2 w TSE MRI (not shown), a Bartolin cyst of $8 \mathrm{~mm}$ in diameter was found on the right side together with the typical pattern of increased signal intensity involving the posterior labial branches of both sides (arrow) on double IR FAT sat _ BB pulse sequences

TSE: Turbo spin echo, MRI: Magnetic resonance imaging, Obt: Obturator internus muscle, Cl: Clitoris, A: Anal canal, Gl: Gluteus max muscle, IR: Inversion-recovery

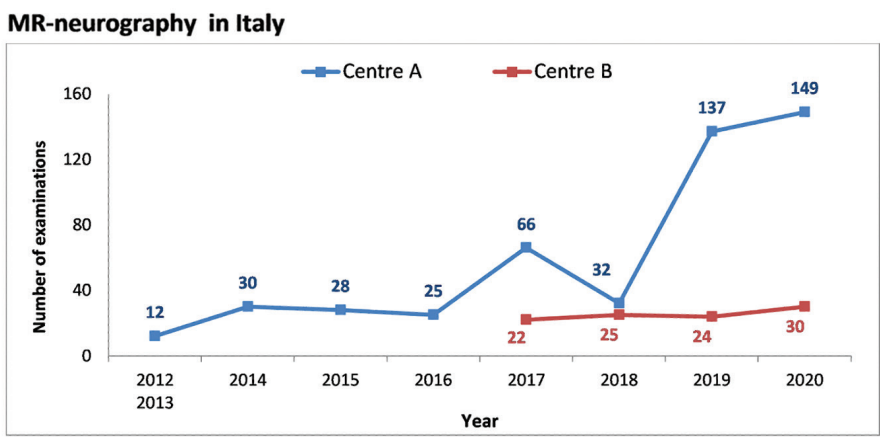

Figure 4. Graph showing the trends of time of the MR-neurography examinations from the beginning of activity in centre A (Monselice, Padua) and centre B (Osimo Stazione, Ancona): total number 479 and 101, respectively. Due to the small number of examinations, the activity of 2012 and 2013 at centre A is cumulative MR: Magnetic resonance

peripheral nervous system providing information concerning morphological alteration of the nerve and surrounding tissues. In addition, it can be very useful in monitoring of the response 
to therapy. At present, MR neurography which uses fat and flow suppression pulse sequences to remove signal from non-neural structures has become available for routine investigation of pudendal nerve pathology. In the future, the use of diffusion weighted imaging sequences, i.e. tractography, which represents the directional motion of intraneural water by colour-coded maps, will provide further information about orientation and degree of architectural organization of the nerve. As such, MR imaging will properly be used to assess also the status of innervated musculature, thus helping in diagnosis, localization of disease and recognition of the degree of nerve damage.

\section{Ethics}

Ethics Committee Approval: Retrospective study.

Informed Consent: For this type of study formal consent is not required.

Peer-review: Externally peer-reviewed.

\section{DISCLOSURES}

Conflict of Interest: The authors have no conflicts of interest.

Financial Disclosure: The authors of the publication did not receive any financial support by any grant/research sponsor.

\section{REFERENCES}

1. Howe FA, Filler AG, Bell BA, et al. Magnetic resonance neurography. Magn Reson Med 1992; 28: 328-38.

2. Filler AG, Kliot M, Howe FA, et al. Application of magnetic resonance neurography in the evaluation of patients with peripheral nerve pathology. J Neurosurg 1996; 85: 299-309.

3. Filler AG, Maravilla KR, Tsuruda JS. MR neurography and muscle MR imaging for image diagnosis of disorders affecting the peripheral nerves and musculature. Neurologic Clinics 2004; 22: 643-82.

4. Kim S, Choi JY, Huh YM, et al. Role of magnetic resonance imaging in entrapment and compressive neuropathy - what, where, and how to see the peripheral nerves on the musculoskeletal magnetic resonance image: part 1. Overview and lower extremity. Eur Radiol 2007; 17: 139-49.

5. Filler AG. Diagnosis and management of pudendal nerves entrapment syndrome: impact of MR neurography and open MRguided injections. Neurosurg Q 2008; 18: 1-6.

6. Mani V, Itskovich VV, Szimtenings M, et al. Rapid extended coverage simultaneous multisection black-blood vessel wall MR imaging. Radiology 2004; 232: 281-8.

7. Piloni V, Bergamasco M, Bregolin F, et al. MR imaging of the pudendal nerve: a one-year experience on an outpatient basis. Pelviperineology 2014; 33:54-9.

8. Slocumb JC. Neurological factors in chronic pelvic pain: trigger points and the abdominal pelvic pain syndrome. Am J Obstet Gynaecol 1984; 149: 536-43.

9. Benson JT, Griffis K. Pudendal neuralgia, a severe pain syndrome. Am J Obstet Gnecol 2005; 192: 1663-8.

10. Chappel KE, Robson MD, Stonebridge-Foster A, et al. Magic angle effects in MR neurography. Am J. Neuroradiol 2004; 431-40.

11. Lee JC, Yang CC, Kronam BG, et al. Neurophysiologic testing in chronic pelvic pain syndrome: a pilot study. Urology 2001; 58: 24650 . 